\title{
Position and Speed Estimation Algorithm for Permanent Magnet Synchronous Machines Considering Nonlinear Magnetic Effects
}

\author{
Matthias Brodatzki* ${ }^{*}$, Jan Richter*, Johannes Kolb ${ }^{\dagger}$ and Michael Braun* \\ * Institute of Electrical Engineering (ETI), Karlsruhe Institute of Technology (KIT), Karlsruhe, Germany \\ $\dagger$ SHARE at KIT, Schaeffler Technologies AG \& Co. KG, Karlsruhe, Germany \\ $\ddagger$ E-Mail: matthias.brodatzki@kit.edu
}

\begin{abstract}
A descriptive, easy to implement approach for the self sensing operation of permanent magnet synchronous machines (PMSM) is presented in this paper. Position and speed identification is performed by solving a nonlinear equation system with a simple, real-time capable algorithm. The approach considers nonlinear magnetic effects by means of flux linkage maps and thus provides reliable position and speed identification in the full speed and torque operating range for stationary and dynamic operation. An experimental proof of functionality is presented.
\end{abstract}

Index Terms-Nonlinear systems, Optimization methods, Permanent magnet machines, Sensorless control

\section{INTRODUCTION}

Dynamic and precise control of electrical machines requires exact knowledge of rotor position and speed. To obtain these values, speed- and position-sensors, attached to the machine shaft, are usually used. Among other drawbacks, these sensors are costly, require space and reduce system reliability [1]. These problems have led to research and publication of numerous position and speed identification methods for various types of electrical machines. The desired information can be extracted from the electromagnetic behavior of the respective machine, thus the methods are also called self sensing [2], [3].

This paper puts a focus on the self sensing control of permanent magnet synchronous machines (PMSM). Two characteristics of the PMSM are most commonly used to determine rotor position and speed, the back electromotive force (EMF) and magnetic anisotropy, which results in rotor angle dependent phase inductances [4]. At high speed, back EMF carries information about rotor speed and position [5]. At low speed, back EMF is very low, respectively non existent at standstill. Low speed self sensing methods rely on the estimation of phase inductances. Therefor, high frequency voltages or currents, so called excitation signals, are injected. The resulting electromagnetic response is measured and allows to identify the phase inductances and thus the present rotor position [2].

Another issue, which has to be handled is, that especially for high power density machines, e.g. in automotive applications, a reduced amount of iron material is used. On the one hand, this reduces costs, but on the other hand these machines show a highly nonlinear behavior due to effects like magnetic saturation, cross-coupling and iron losses [6]-[9]. These nonlinearities can render current control and self sensing position estimation impossible with common approaches.

All mentioned characteristics of the PMSM can be described by means of a mathematical machine model. The presented algorithm compares measured electrical quantities with calculated values obtained from a machine model. The present rotor position angle and speed is identified by minimizing their differences. In contrast to similar approaches [10], a nonlinear model, supported by flux linkage maps, is used. Hence, magnetic saturation and cross-coupling are covered, allowing dynamic operation in the complete current range.

The underlying machine model is briefly described in section II and represents the base for the nonlinear identification equations derived in section III together with the identification algorithm itself. Experimental results are shown and discussed in section IV.

\section{PMSM MODEL}

Although a complex machine model could be used with the presented method, this paper will only cover a fundamental model for the sake of clarity and to investigate the influence of model errors. Slotting effects, iron losses and temperature dependencies of machine parameters are neglected.

Electrical machines are usually modelled using a rotor or magnetic flux aligned reference frame. It allows the distinct description of direct- and quadrature-components of all physical values. The PMSM fundamental model in the rotor aligned reference frame is given by equations (1) and (2).

$$
\begin{aligned}
& v_{\mathrm{d}}=R i_{\mathrm{d}}+\frac{\mathrm{d} \psi_{\mathrm{d}}\left(i_{\mathrm{d}}, i_{\mathrm{q}}\right)}{\mathrm{d} t}-\omega \psi_{\mathrm{q}}\left(i_{\mathrm{d}}, i_{\mathrm{q}}\right) \\
& v_{\mathrm{q}}=R i_{\mathrm{q}}+\frac{\mathrm{d} \psi_{\mathrm{q}}\left(i_{\mathrm{d}}, i_{\mathrm{q}}\right)}{\mathrm{d} t}+\omega \psi_{\mathrm{d}}\left(i_{\mathrm{d}}, i_{\mathrm{q}}\right)
\end{aligned}
$$

In the fundamental machine model, the resulting $\mathrm{d}$ - and q-flux linkages $\psi_{\mathrm{d}}$ and $\psi_{\mathrm{q}}$ and thus the inner torque only depend on the respective current components and not on the rotor angle.

The phase resistance $R$ can be measured and is considered constant. The machine is assumed to have symmetric phase resistances, so $R$ is valid in any reference frame. The current-dependent flux linkages $\psi_{\mathrm{d}}\left(i_{\mathrm{d}}, i_{\mathrm{q}}\right)$ and 


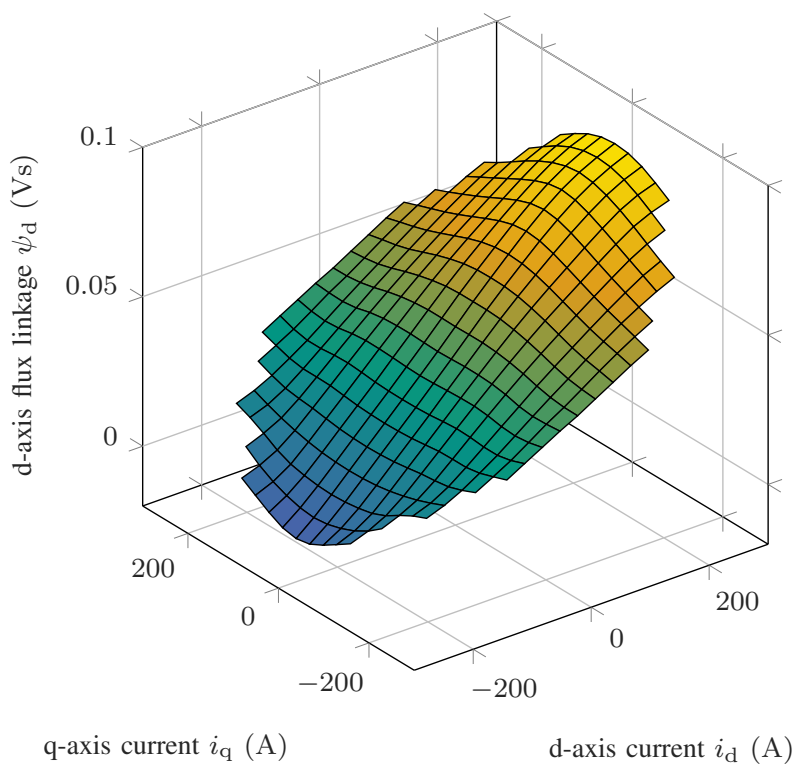

(a)

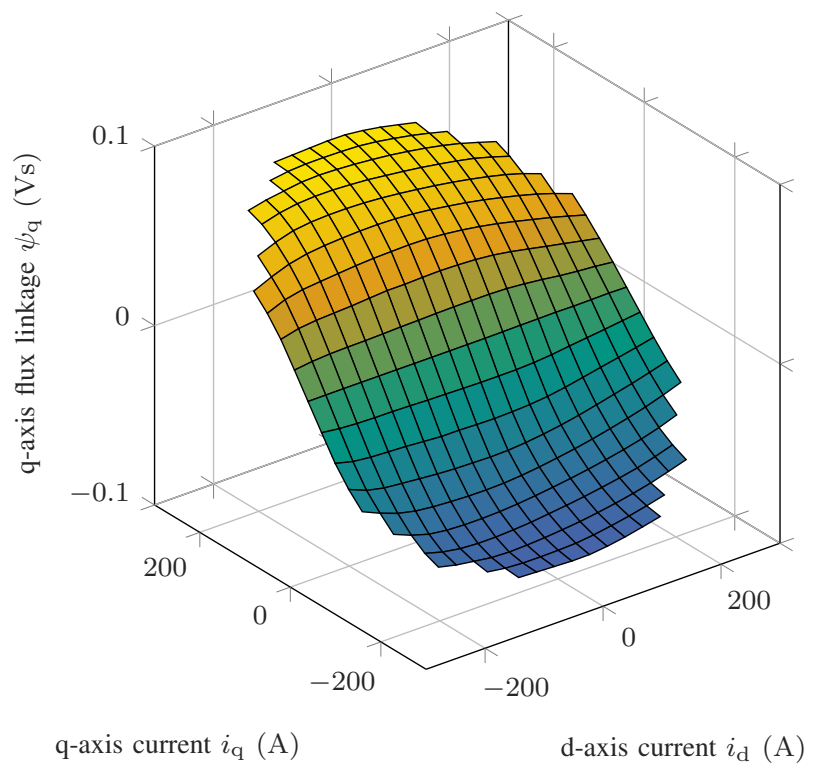

(b)

Fig. 1: Flux linkage maps $\psi_{\mathrm{d}}\left(i_{\mathrm{d}}, i_{\mathrm{q}}\right)$ and $\psi_{\mathrm{q}}\left(i_{\mathrm{d}}, i_{\mathrm{q}}\right)$

$\psi_{\mathrm{q}}\left(i_{\mathrm{d}}, i_{\mathrm{q}}\right)$ can be acquired [11] and are stored as flux linkage maps like shown in figs. $1 \mathrm{a}$ and $1 \mathrm{~b}$. By means of these maps, nonlinear magnetic effects can be considered by the control method or in this case by the identification algorithm.

To obtain the rotor aligned reference frame values, the Park-transformation must be applied to the measured phase values. For this transformation, the present rotor position angle $\gamma$ has to be known. If there is no position sensor available, rotor position and angular speed can still be determined using the machine model itself. To clarify this, the voltage equations from above will be rewritten in the stator oriented reference frame.

$$
\begin{aligned}
& v_{\alpha}=R i_{\alpha}+\left.\frac{\mathrm{d} \psi_{\alpha}\left(i_{\alpha}, i_{\beta}, \gamma\right)}{\mathrm{d} t}\right|_{\gamma=\text { const. }}-\omega \psi_{\beta}\left(i_{\alpha}, i_{\beta}, \gamma\right) \\
& v_{\beta}=R i_{\beta}+\left.\frac{\mathrm{d} \psi_{\beta}\left(i_{\alpha}, i_{\beta}, \gamma\right)}{\mathrm{d} t}\right|_{\gamma=\text { const. }}+\omega \psi_{\alpha}\left(i_{\alpha}, i_{\beta}, \gamma\right)
\end{aligned}
$$

If $\gamma$ and $\omega$ are unknown, this is a nonlinear equation system with two equations and two variables. Currents $i_{\alpha}$ and $i_{\beta}$ are measured, voltages $v_{\alpha}$ and $v_{\beta}$ can be measured or obtained from a suitable converter model. Machine resistances and flux linkages are known, whereby the latter have to be transformed by (5), since they are usually stored as current dependent $\mathrm{d}$ - and q-components.

$$
\underline{\psi}_{\alpha \beta}\left(\underline{i}_{\alpha \beta}, \gamma\right)=\underline{\psi}_{\mathrm{dq}}\left(\underline{i}_{\alpha \beta} \cdot e^{-\mathrm{j} \gamma}\right) \cdot e^{\mathrm{j} \gamma}
$$

Obviously, the problem of self sensing lies within the solution of equation system (3)-(4). Unfortunately, this is not trivial, since no closed-form solution exists. The presented algorithm aims at solving this equation system to obtain precise values of rotor position and speed even under highly nonlinear conditions.

\section{Position AND SPEED IDENTIFICATION}

The machine voltage equations (3) and (4) can be used to calculate expected voltages for measured currents $i_{\alpha}, i_{\beta}$ and any arbitrary rotor position and speed. When the correct present values of $\gamma$ and $\omega$ are inserted, the calculated and measured voltages $v_{\alpha}$ and $v_{\beta}$ are equal. Thus, minimizing the difference between measurement and model through variation of the model variables $\gamma$ and $\omega$ leads to a correct solution. The resulting equations, whose zeros have to be found are (6) and (7).

$$
\begin{aligned}
\Delta v_{\alpha}(\gamma, \omega)= & v_{\alpha} \\
& -R i_{\alpha}+\left.\frac{\mathrm{d} \psi_{\alpha}\left(i_{\alpha}, i_{\beta}, \gamma\right)}{\mathrm{d} t}\right|_{\gamma=\text { const. }} \\
& -\omega \psi_{\beta}\left(i_{\alpha}, i_{\beta}, \gamma\right) \\
\Delta v_{\beta}(\gamma, \omega)= & v_{\beta} \\
& -R i_{\beta}+\left.\frac{\mathrm{d} \psi_{\beta}\left(i_{\alpha}, i_{\beta}, \gamma\right)}{\mathrm{d} t}\right|_{\gamma=\text { const. }} \\
& +\omega \psi_{\alpha}\left(i_{\alpha}, i_{\beta}, \gamma\right)
\end{aligned}
$$

Plotting these two-dimensional functions leads to voltage difference maps, which can be seen in figs. $2 \mathrm{a}$ and $2 \mathrm{~b}$ for one electrical rotation and the maximum speed range of a reference machine. For both voltage components, lines of zero voltage difference can be found. These lines indicate, that for the given parameters $\gamma$ and $\omega$, the measured and calculated voltages are equal. Assuming an ideal machine model and phase voltage determination, the rotor position and speed can be found, when measured and calculated voltages are equal for both voltage components. This is the case at the intersections of these lines, as shown in fig. 2c.

Since the voltage equations are ambiguous for an angle shift of $180^{\circ}$ and inverse speed, there will always be two intersections, which is a general problem of self sensing. 
Additional mechanical constraints allow the elimination of the false solution: for high speeds it can be assumed that the machine will not reach reverse speed between two identification steps. For low speeds, the rotor will not rotate $180^{\circ}$ in the short period of time. Nevertheless, the ambiguity must be resolved at the beginning of operation using a suitable initialization routine [12]-[14].

\section{A. Identification Algorithm Implementation}

Consequently, self sensing is performed by precisely finding the intersection of functions $\Delta v_{\alpha}(\gamma, \omega)=0$ and $\Delta v_{\beta}(\gamma, \omega)=0$. For this non-linear problem, an efficient algorithm is presented, which can be used on a real time control system, i.e. to dynamically find rotor position and speed in every control cycle.

The algorithm is visualized in fig. 3. The first step is to define an area in which the intersection is expected, as shown in fig. 3a. The area should be delimited by at least the feasible speed and angle range, that can be reached during the given control period. The center point of the area can be determined from the past values of speed and angle and assuming constant rotation between two control periods.

The values of $\Delta v_{\alpha}$ and $\Delta v_{\beta}$ are calculated for the corners of the searching area. Afterwards, for $\Delta v_{\alpha}$ as well as $\Delta v_{\beta}$, zero crossings along the borders of the searching area are identified by linear approximation between the corner values. Connecting the zero crossing points on the borders results in approximated $\Delta v_{\alpha}=0$ and $\Delta v_{\beta}=0$ lines, as shown in figs. $3 \mathrm{~b}$ and $3 \mathrm{c}$.

The intersection of these lines can be used as starting point of the next iteration with smaller searching area, as shown in fig. 3d. As depicted in figs. $3 \mathrm{e}$ and $3 \mathrm{f}$, the solution becomes more precise with each iteration, because the error caused by linearization becomes smaller. The more nonlinear a machine is, the more iterations can be necessary. For the machine used in this paper, three iterations have been sufficient.

\section{B. Low Speed Operation}

A benefit of the presented method is that it can be used in the full speed range up from standstill. Yet, it has to be ensured, that the machine remains observable. At low speed operation back EMF becomes small, respectively vanishes at standstill. When in stationary operation, only an ohmic voltage drop due to the phase currents can be measured. The phase resistances are usually symmetric and do not carry any information about rotor position.

To overcome this problem, a general approach is to inject high frequency excitation currents. The resulting phase voltages, induced due to change of flux linkages, contain rotor position information. This is because phase inductances depend on the rotor angle for suitable PMSMs, caused by magnetic anisotropy $\left(l_{\mathrm{dd}}=\mathrm{d} \psi_{\mathrm{d}} / \mathrm{d} i_{\mathrm{d}} \neq\right.$ $\left.l_{\mathrm{qq}}=\mathrm{d} \psi_{\mathrm{q}} / \mathrm{d} i_{\mathrm{q}}\right)$. Anisotropy is a prerequisite for low speed operation with the presented approach as for most self sensing methods.

The effect of excitation signal injection can be shown visually in a descriptive way. The situation resulting from

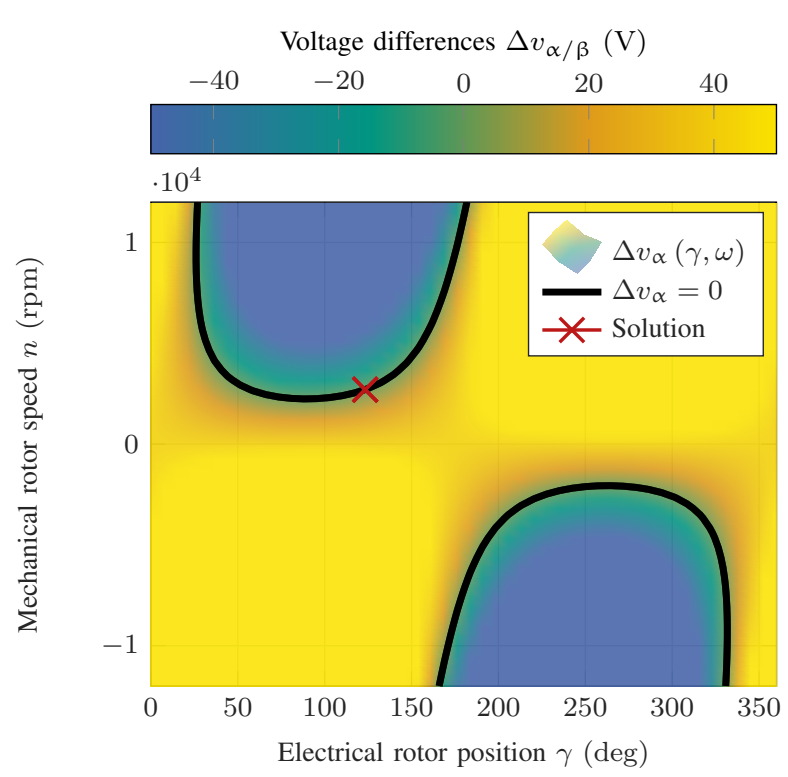

(a) Height map of $\Delta v_{\alpha}(\gamma, \omega)$ with height lines indicating $\Delta v_{\alpha}=0$

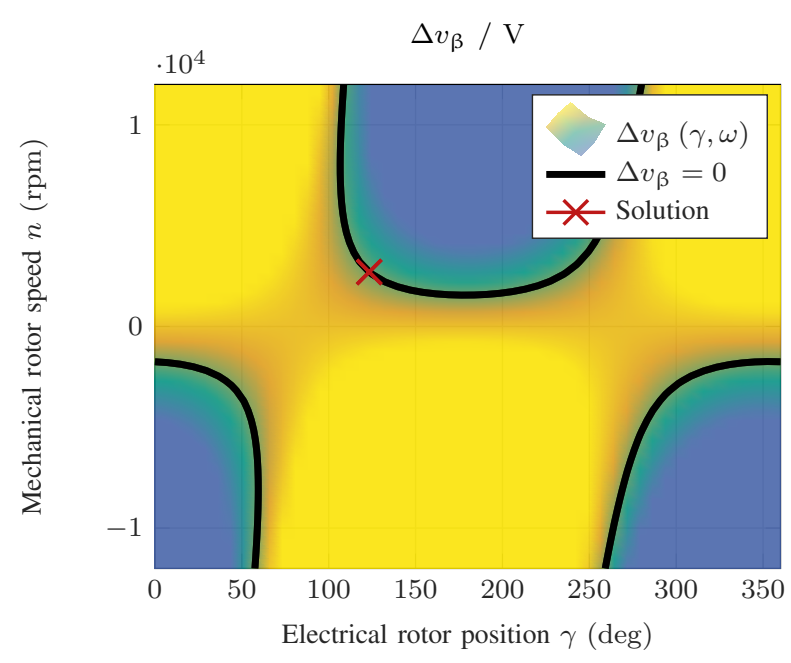

(b) Height map of $\Delta v_{\beta}(\gamma, \omega)$ with height lines indicating $\Delta v_{\beta}=0$

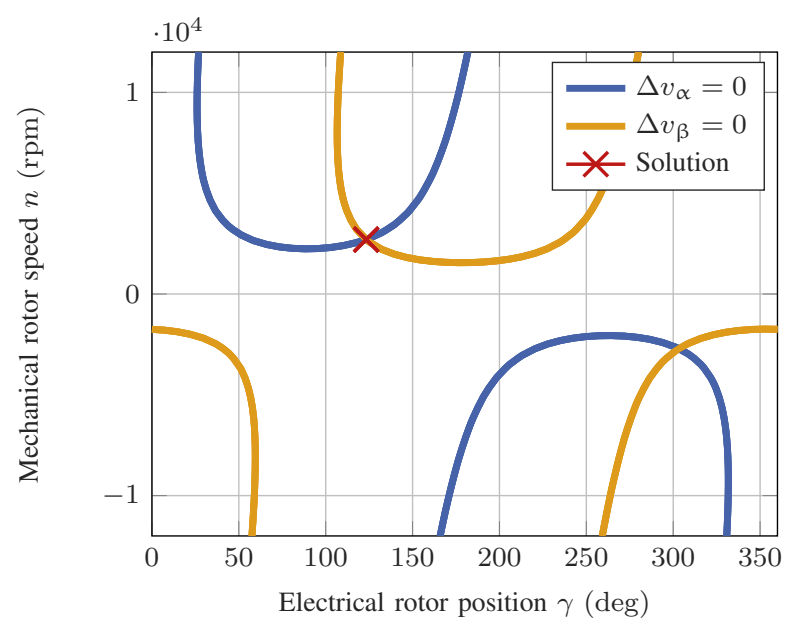

(c) Coordinates, at which measured and calculated voltages equal, are feasible solutions. Thus, the rotor position and speed can be found at an intersection of the lines defined by $\Delta v_{\alpha}=0$ and $\Delta v_{\beta}=0$.

Fig. 2: Graphical representation of the approach. The red cross shows the correct solution. 


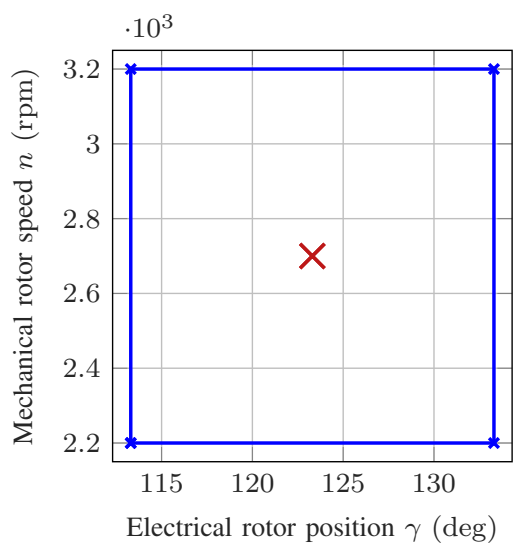

(a) Step 1: Definition of a feasible search area

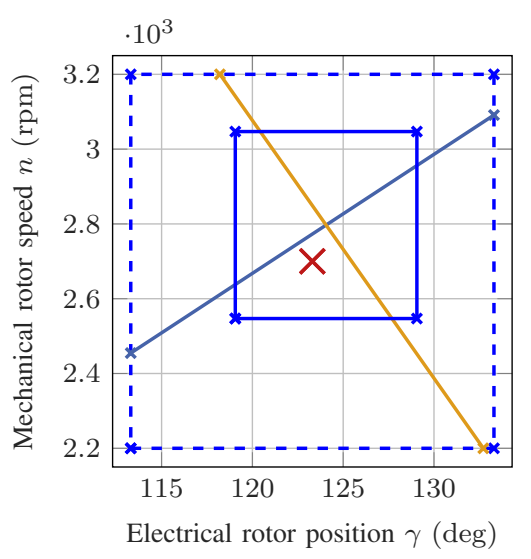

(d) Step 4: Definition of a new, smaller search area around the found intersection

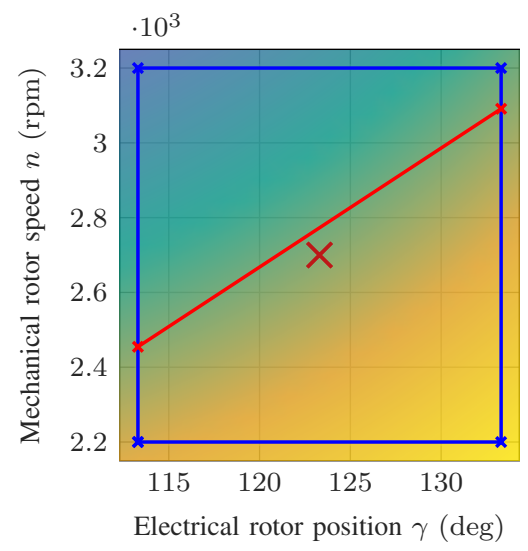

(b) Step 2: Linear approximation of the $\Delta v_{\alpha}=0$ line using the search area corner values of $\gamma$ and $\omega$ in (6)

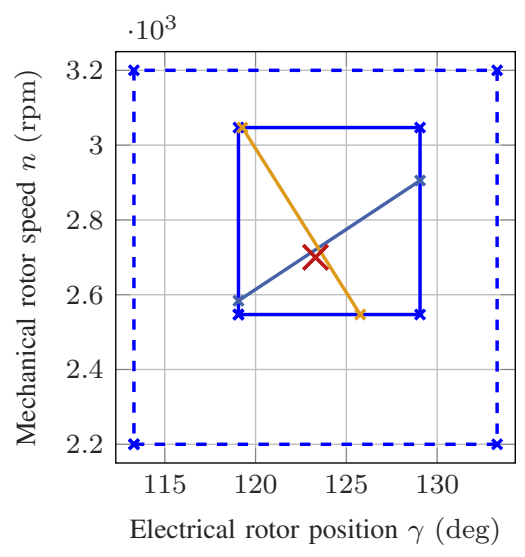

(e) Iteration of the described steps. With each iteration, errors caused by nonlinearities become smaller.

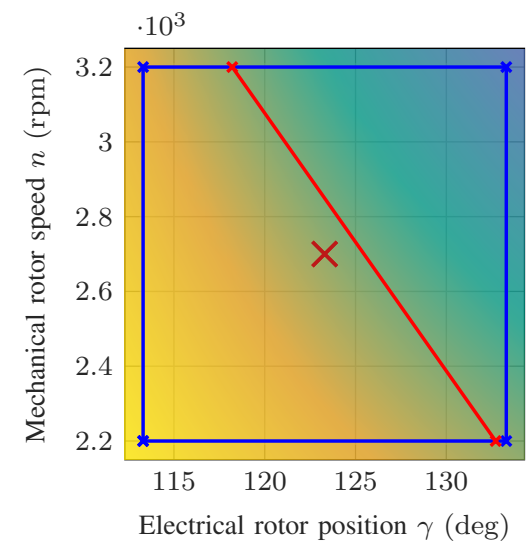

(c) Step 3: Linear approximation of the $\Delta v_{\beta}=0$ line using the search area corner values of $\gamma$ and $\omega$ in (7)

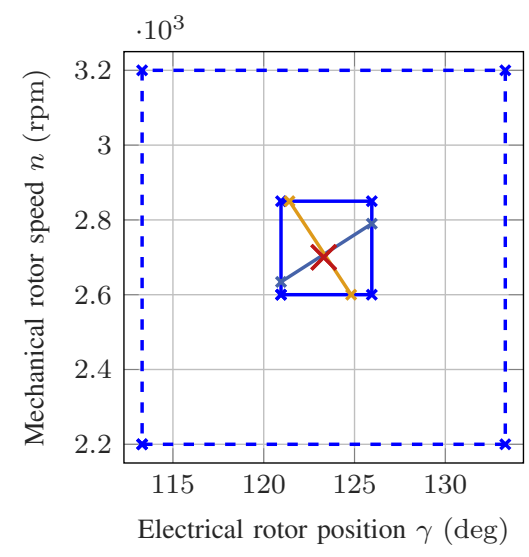

(f) After the last iteration, the found solution should be satisfying. The number of necessary iterations depends on the machine.

Fig. 3: Visualization of the identification algorithm. The red cross marks the correct solution of the identified pair of $\gamma$ and $n$.

vanishing EMF is depicted in fig. 4a. The zero lines of the identification equations have no intersections but overlap for most values of $\gamma$. Leading to an infinite amount of solutions of the equation system, the rotor position and speed can't be determined anymore. During the injection of high frequency currents, distinct intersections occur as can be seen in fig. 4b. Thus, a reliable rotor position and speed identification is possible again.

To allow operation in the full speed range, known approaches usually switch between different identification methods for high- and low-speed. With the presented approach, it is sufficient to inject a high frequency current below a certain speed limit, because the current-slope induced voltages are also modelled by the machine voltage equations and considered in (6) and (7). The high frequency signal can be superimposed on the reference of the current controller.

A block diagram of an exemplary current controlled electrical drive system, including the presented approach for rotor position and speed identification, is shown in fig. 5. The same structure is valid for the experimental implementation in the following chapter.

\section{EXPERIMENTAL RESULTS}

For experimental validation, several measurements are performed with the setup depicted in fig. 6. Two equal and mechanically coupled automotive PMSMs are used. The machine parameters are listed in table I. One machine is speed-controlled and a reference position sensor provides position and speed information. The second machine is operated with current control and the presented approach is used to identify its rotor speed and position. The identification is performed in closed loop, so no information from a position sensor is used for the current transformation and control as shown in fig. 5. The identified rotor position is filtered by linear regression to reduce the influence of current and voltage measurement noise.

A Si-IGBT inverter is used with a switching frequency of $f_{\mathrm{S}}=8 \mathrm{kHz}$ and a dc-link voltage of $v_{\mathrm{DC}}=250 \mathrm{~V}$. The dc-link and output voltages of the inverter and the phase currents are measured. The identification algorithm is fully executed during every switching period on a Texas Instruments TMS320C6748 based real-time signal processing system [15]. Thus, every $125 \mu \mathrm{s}$ a new rotor position and speed value is available. 


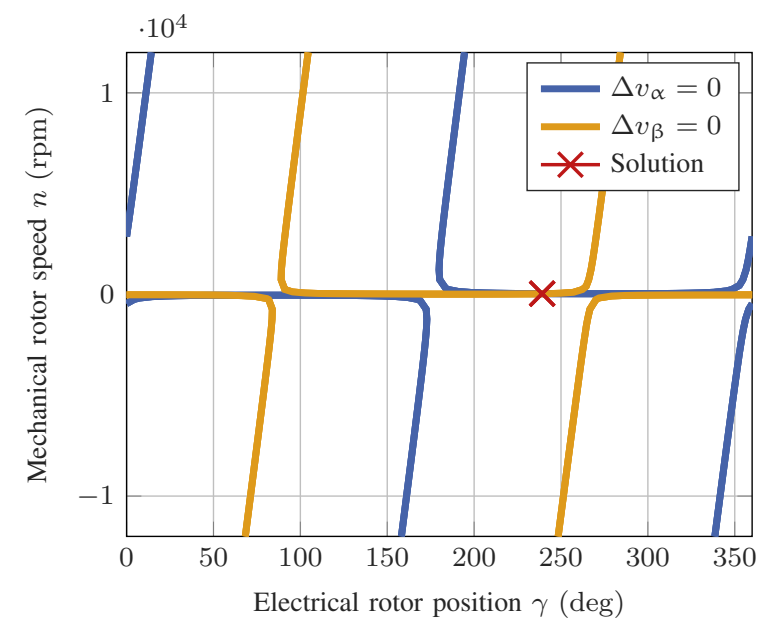

(a) When EMF vanishes due to low speed, the zero-lines overlap and no solution can be found.

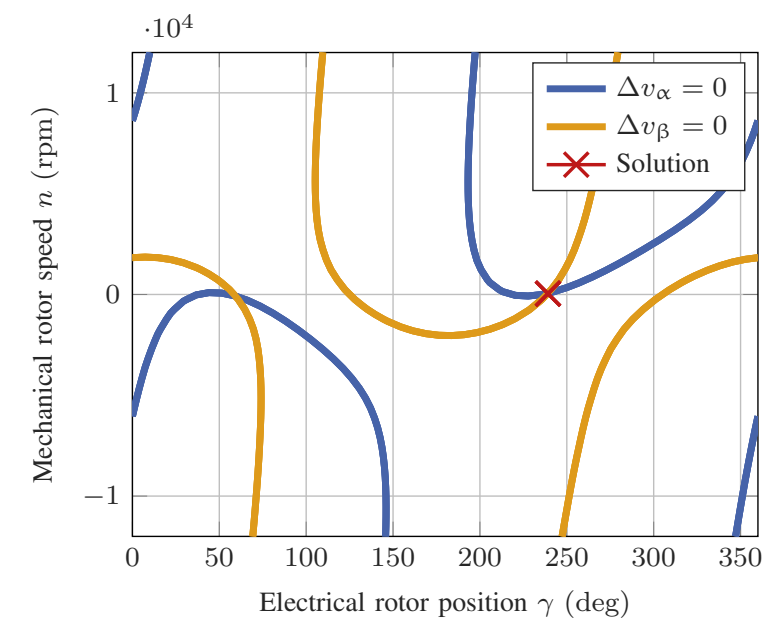

(b) The injection of high frequency currents leads to restored feasability. Distinct intersections of the zero-lines occur again.

Fig. 4: Effect of high frequency current injection at low speed and standstill

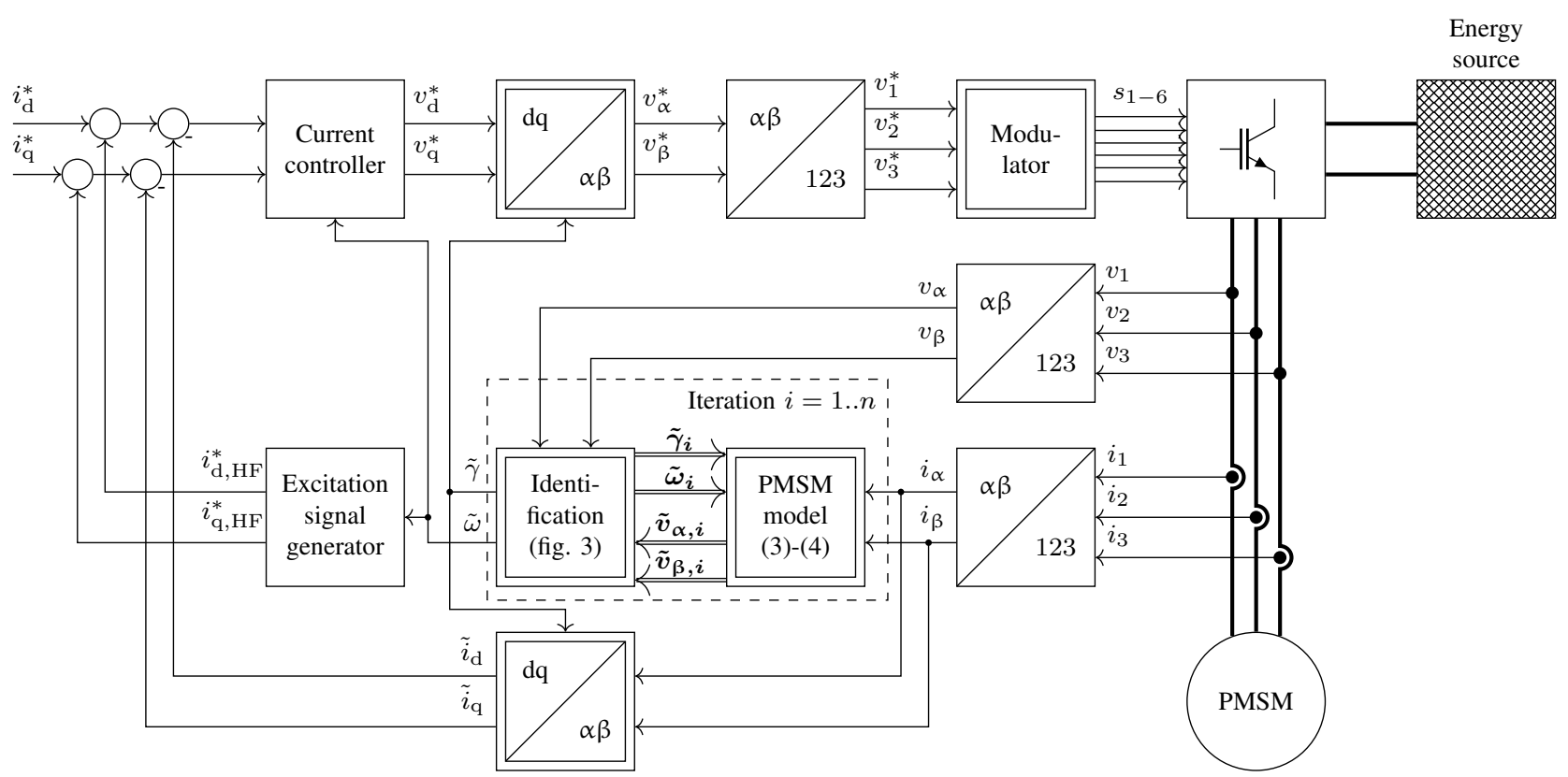

Fig. 5: Block diagram of an electrical drive system with the presented position and speed identification

TABLE I: MAChine PARAMETERS

\begin{tabular}{lll}
\hline Parameter & Symbol & Value \\
\hline Rated power & $P_{\mathrm{N}}$ & $35 \mathrm{~kW}$ \\
Rated torque & $T_{\mathrm{N}}$ & $80 \mathrm{Nm}$ \\
Rated phase voltage & $V_{\mathrm{ph}, \mathrm{N}}$ & $110 \mathrm{~V}$ \\
Rated current & $I_{\mathrm{N}}$ & $160 \mathrm{~A}$ \\
Rated speed & $n_{\mathrm{N}}$ & $4000 \mathrm{rpm}$ \\
Pole pairs & $p$ & 4 \\
Phase inductance d-component & $L_{\mathrm{d}}$ & $120 \mu \mathrm{H}$ to $210 \mu \mathrm{H}$ \\
Phase inductance q-component & $L_{\mathrm{q}}$ & $180 \mu \mathrm{H}$ to $550 \mu \mathrm{H}$ \\
\hline
\end{tabular}

When the rotor speed is low $(-300 \mathrm{rpm} \leq n \leq$ $300 \mathrm{rpm})$, a high frequency current is injected with $f_{\mathrm{HF}}=$ $4 \mathrm{kHz}, \hat{i}_{\mathrm{d}, \mathrm{HF}}=0.5 \mathrm{~A}$ and $\hat{i}_{\mathrm{q}, \mathrm{HF}}=5 \mathrm{~A}$.

To validate the algorithm at different rotor speeds, a speed reference ramp from $-\frac{1}{2} n_{\mathrm{n}}$ to $\frac{1}{2} n_{\mathrm{n}}$ is applied to the speed controlled machine as can be seen in fig. 9a. The inner torque reference of the current controlled (self sensing) machine is set to $T_{\mathrm{i}, \mathrm{w}}=T_{\mathrm{N}}$ respectively $T_{\mathrm{i}, \mathrm{w}}=\frac{1}{2} T_{\mathrm{N}}$. The identified rotor speed and position are compared to the values of a reference position sensor. Resulting errors are shown in figs. $9 \mathrm{~b}$ and $9 \mathrm{c}$. The estimation errors stay low, verifying the stability of the presented method. Also for standstill and low speeds, the identification is reliable.

The speed and load dependent identification error, which can be seen, occurs because iron losses were neglected in the machine model. Iron losses and the resulting model errors become larger at higher electric angular velocity and higher magnetic flux. Also for low speeds, when high frequency currents are injected, iron losses distort the machine model and lead to increased estimation errors. As can be seen, position error is constant in the speed range $-300 \mathrm{rpm}$ to $300 \mathrm{rpm}$. This is the case, 


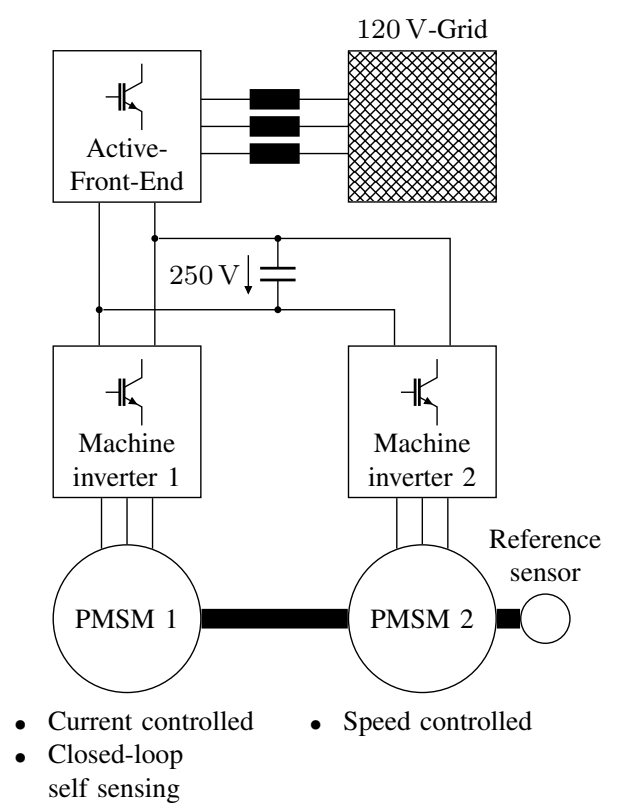

Fig. 6: Schematic of the experimental setup

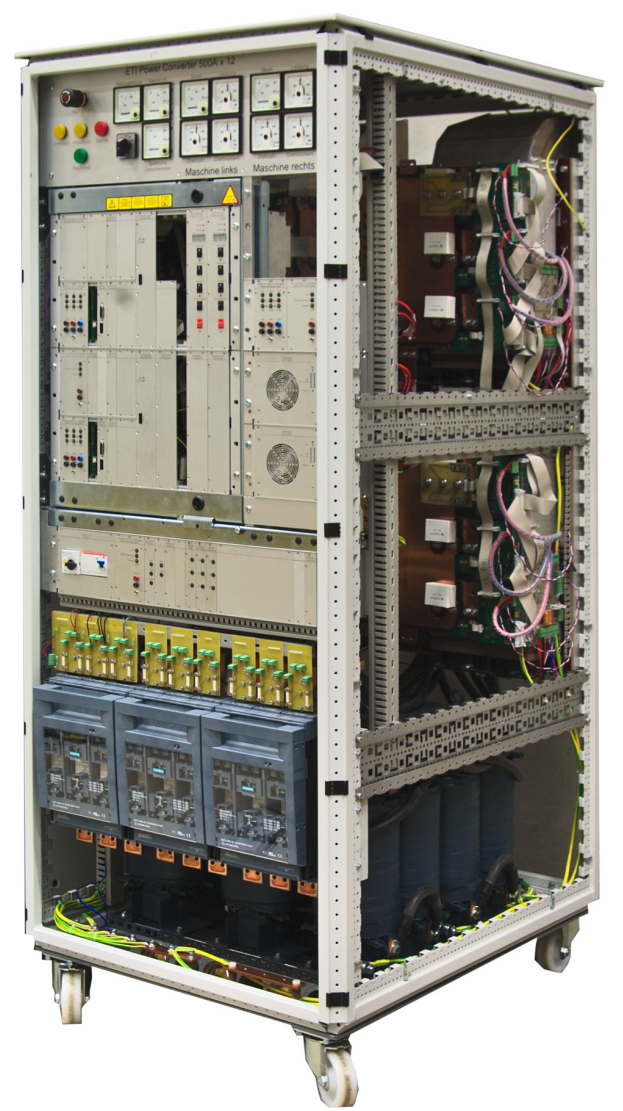

Fig. 7: Photography of the used inverter cabinet. It consists of an active-front-end inverter, two machine inverters and a real-time signal processing system.

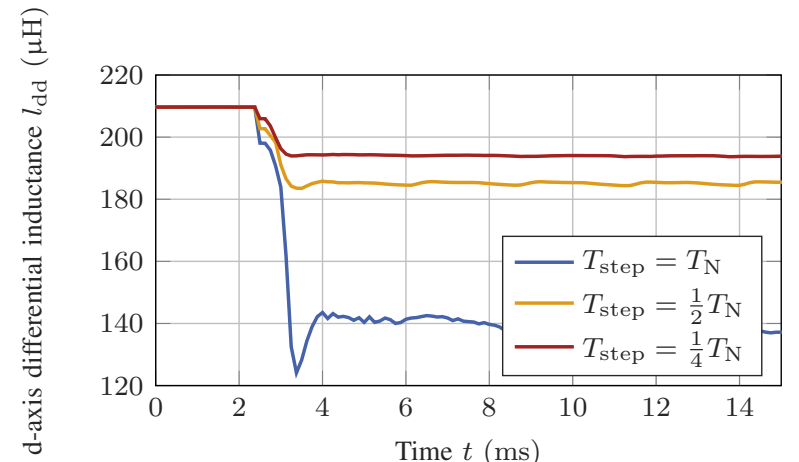

(a)

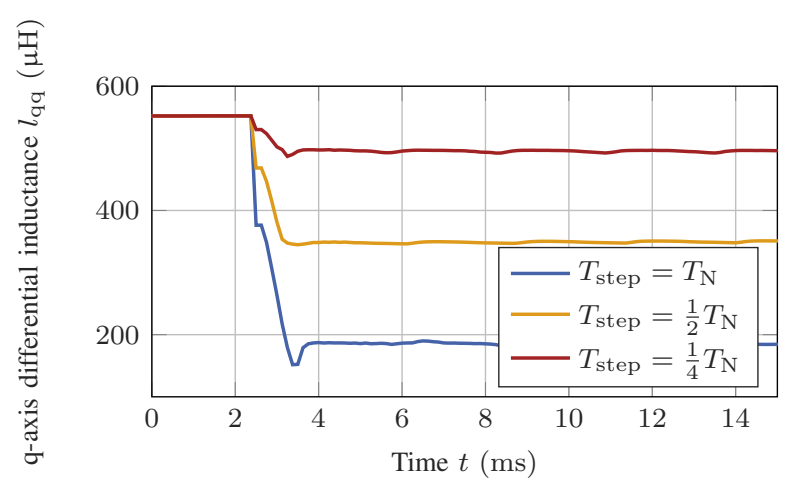

(b)

Fig. 8: Variation of the differential phase inductances during different torque reference steps as measure of the machine nonlinearities

because losses caused by high frequency current injection are dominant at low speeds, since the d- and q-axis fluxlinkage variations also generate losses in the rotor iron and magnets as opposed to flux-linkage variations due to rotation. The described errors can be reduced, when iron losses are considered in the machine model.

To validate the dynamics and the robustness against nonlinearities, large torque reference steps are applied to the self sensing machine, while the speed-controlled machine reference is set to $1000 \mathrm{rpm}$ respectively $0 \mathrm{rpm}$.

The estimated and real inner torque may differ due to incorrect position estimation. Therefore, the inner torque (8) is calculated with the estimated as well as real dand q-currents, correctly transformed with the position sensor output angle. The difference of both values is the resulting inner torque error. For highly dynamic operation, this error should stay low.

$$
T=\frac{3}{2} p\left(\psi_{\mathrm{d}} \cdot i_{\mathrm{q}}-\psi_{\mathrm{q}} \cdot i_{\mathrm{d}}\right)
$$

The results can be seen in figs. 10 and 11. For both rotor speeds, the real inner torque (figs. 10a and 11a), the position identification error (figs. $10 \mathrm{~b}$ and $11 \mathrm{~b}$ ) and the inner torque error (figs. 10c and 11c) are provided. As a measure for the occurring nonlinearities, the differential inductances $l_{\mathrm{dd}}$ and $l_{\mathrm{qq}}$ are shown in fig. 8 .

The resulting position estimation error stays below $8^{\circ}$ and allows dynamic, stable and precise current control with a torque error below $5 \%$. 


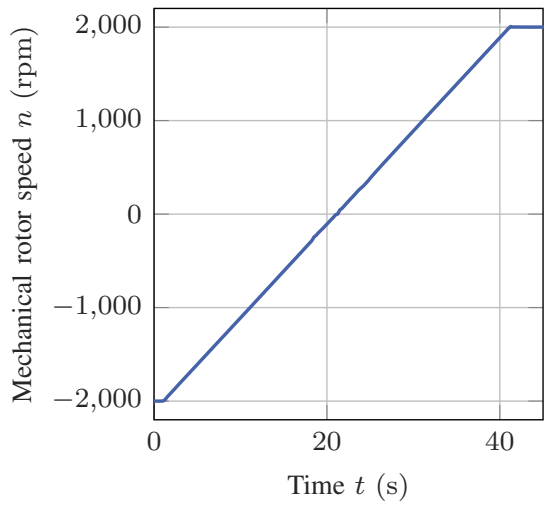

(a) Measured speed

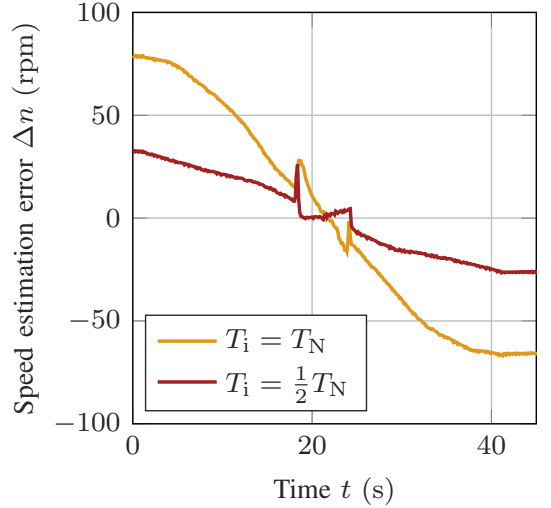

(b) Absolute estimation error of $n$

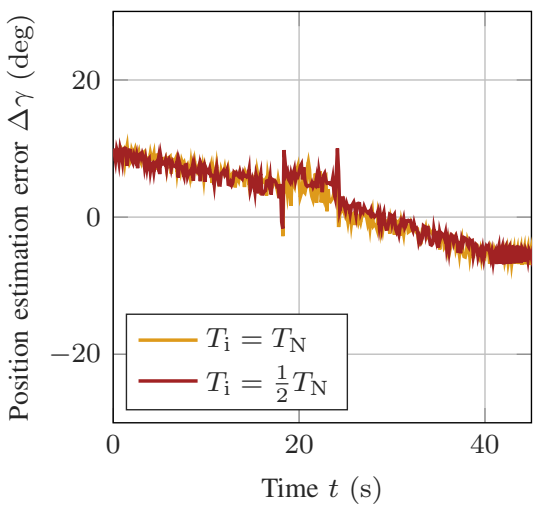

(c) Absolute estimation error of $\gamma$

Fig. 9: Mechanical speed and electrical angle identification errors during a speed reference reversal at nominal torque and half nominal torque

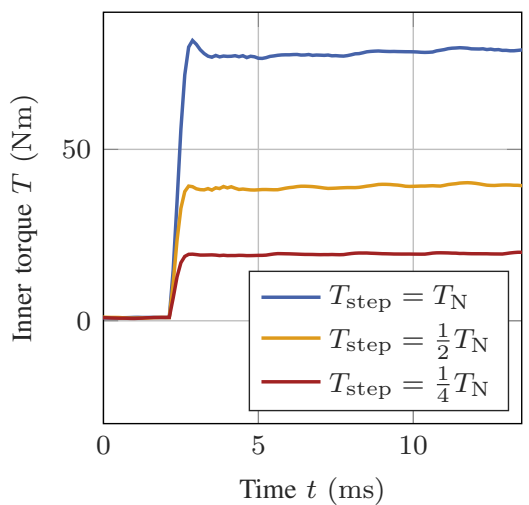

(a) Inner torque of the self sensing machine during different torque reference steps

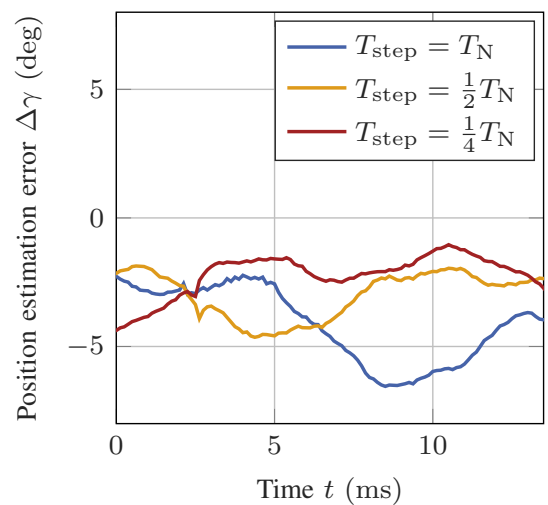

(b) Absolute estimation error of $\gamma$ during different torque reference steps

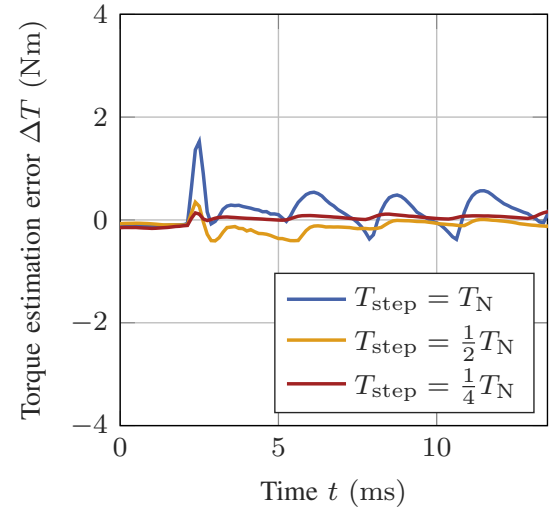

(c) Absolute inner torque error during different torque reference steps

Fig. 10: Dynamic control performance and angle estimation error during different torque reference steps at $n=1000 \mathrm{rpm}$

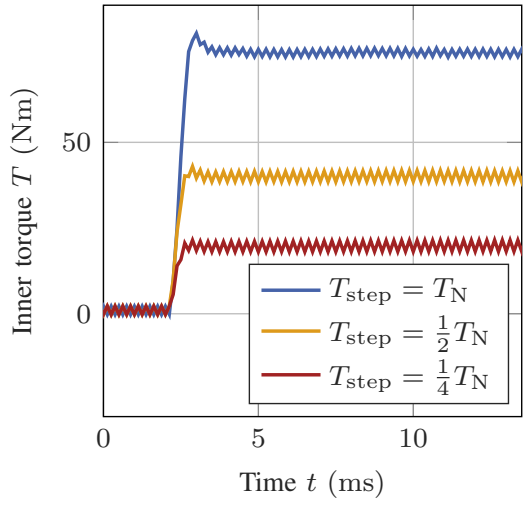

(a) Inner torque of the self sensing machine during different torque reference steps

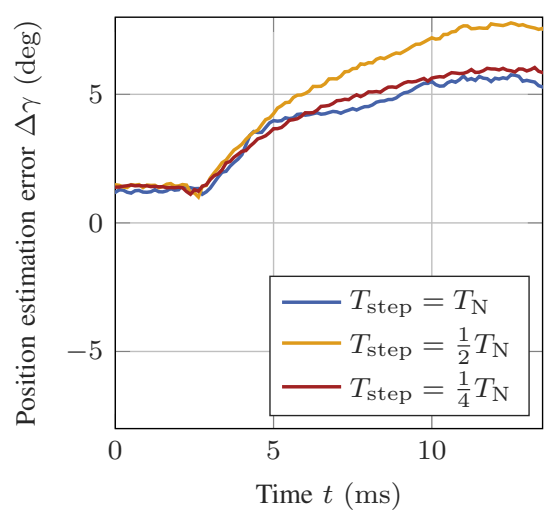

(b) Absolute estimation error of $\gamma$ during different torque reference steps

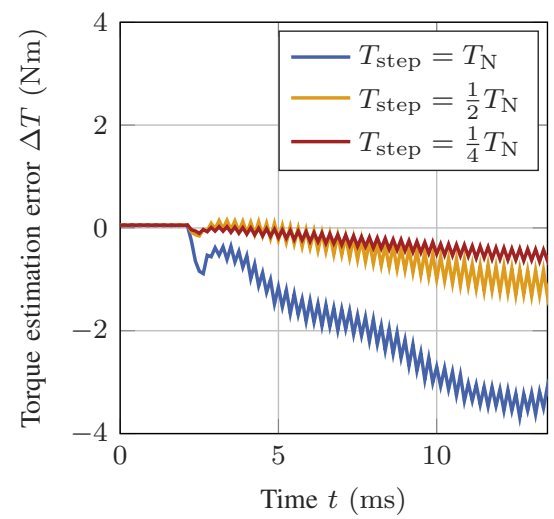

(c) Absolute inner torque error during different torque reference steps

Fig. 11: Dynamic control performance and angle estimation error during different torque reference steps at $n=0 \mathrm{rpm}$ 


\section{CONCLUSION}

A descriptive, easily implementable rotor position and speed identification algorithm for nonlinear PMSMs is presented. The problem of position and speed identification is reduced to zero-crossing determinations and an intersection detection of two lines. Though a simple approach, it provides stable and reliable results in the full operating range of a PMSM. Further, the derived equations provide a depiction of self sensing and can help to understand the problems occurring in some operating points, such as low speed.

The provided iterative algorithm can be calculated on a real time control system. When supported by a nonlinear machine model, it can perform self sensing position and speed identification for highly nonlinear machines in the full operating range, even during dynamic operation. This has been proven by experiment. When using an expanded machine model, e.g. considering iron losses or slotting effects, even more precise results can be expected.

\section{REFERENCES}

[1] I. Hahn, "Differential magnetic anisotropy - prerequisite for rotor position detection of pm-synchronous machines with signal injection methods," in 2010 First Symposium on Sensorless Control for Electrical Drives, 2010, pp. 40-49. [Online]. Available: $\mathrm{http} / / /$ ieeexplore.ieee.org/stamp/stamp.jsp?arnumber $=5542803$

[2] F. Briz and M. Degner, "Rotor position estimation," IEEE Industrial Electronics Magazine, vol. 5, no. 2, pp. 24-36, 2011.

[3] R. Bojoi, M. Pastorelli, J. Bottomley, P. Giangrande, and C. Gerada, "Sensorless control of pm motor drives - a technology status review," in 2013 IEEE Workshop on Electrical Machines Design, Control and Diagnosis (WEMDCD), 2013, pp. 168-182. [Online]. Available: http://ieeexplore.iee.. org/stamp/stamp.jsp?arnumber $=6525177$

[4] P. P. Acarnley and J. F. Watson, "Review of positionsensorless operation of brushless permanent-magnet machines," IEEE Transactions on Industrial Electronics, vol. 53, no. 2, pp. 352-362, 2006.

[5] F. Genduso, R. Miceli, C. Rando, and G. R. Galluzzo, "Back emf sensorless-control algorithm for high-dynamic performance pmsm," IEEE Transactions on Industrial Electronics, vol. 57, no. 6, pp. 2092-2100, 2010.
[6] J. Graus, A. Boehm, and I. Hahn, "Influence of stator saturation on the differential inductances of pm-synchronous machines with concentrated winding," in 3rd IEEE International Symposium on Sensorless Control for Electrical Drives (SLED 2012), 2012, pp. 1-7. [Online]. Available: http://ieeexplore.ieee.org/stamp/stamp.jsp?arnumber=6422809

[7] L. Alberti, N. Bianchi, and S. Bolognani, "Comparison of different synchronous machines for sensorless drives," in IECON 2013 - 39th Annual Conference of the IEEE Industrial Electronics Society, 2013, pp. 8220-8226. [Online]. Available: http://ieeexplore.ieee.org/stamp/stamp.jsp?arnumber $=6700509$

[8] N. Bianchi, S. Bolognani, and M. Zigliotto, "Design hints of an ipm synchronous motor for an effective position sensorless control," in IEEE 36th Conference on Power Electronics Specialists, 2005. IEEE, 2005, pp. 1560-1566.

[9] J. Richter and M. Doppelbauer, "Control and mitigation of current harmonics in inverter-fed permanent magnet synchronous machines with non-linear magnetics," IET Power Electronics, vol. 9, no. 10, pp. 2019-2026, 2016.

[10] Y. Sun, M. Preindl, S. Sirouspour, and A. Emadi, "Unified wide-speed sensorless scheme using nonlinear optimization for ipmsm drives," IEEE Transactions on Power Electronics, vol. 32, no. 8, pp. 6308-6322, 2017.

[11] J. Richter, A. Dollinger, and M. Doppelbauer, "Iron loss and parameter measurement of permanent magnet synchronous machines," in 2014 International Conference on Electrical Machines (ICEM). IEEE, 02.09.2014 05.09.2014, pp. 1635-1641.

[12] S. Zossak, M. Stulraiter, P. Makys, and M. Sumega, "Initial position detection of pmsm," in 2018 IEEE 9th International Symposium on Sensorless Control for Electrical Drives (SLED). IEEE, 9/13/2018 - 9/14/2018, pp. 12-17.

[13] V. Manzolini, M. Morandin, and S. Bolognani, "D-axis polarity detection for ipm synchronous motor drives by high frequency voltage injection," in IECON 2016 - 42nd Annual Conference of the IEEE Industrial Electronics Society. IEEE, 23.10.2016 - 26.10.2016, pp. 4325-4330.

[14] M. Seilmeier and B. Piepenbreier, "Initial start-up and magnet polarity estimation method for hf test current injection based sensorless control of pmsm," in 2014 16th International Power Electronics and Motion Control Conference and Exposition. IEEE, 21.09.2014 - 24.09.2014, pp. 59-64.

[15] C. Axtmann, M. Boxriker, and M. Braun, "A custom, highperformance real time measurement and control system for arbitrary power electronic systems in academic research and education," in 2016 18th European Conference on Power Electronics and Applications (EPE'16 ECCE Europe). IEEE, 05.09.2016 - 09.09.2016, pp. 1-7. 